\title{
Inverse method for attribution of climate change
}

\author{
Meelis J. Zidikheri ${ }^{1} \quad$ Jorgen S. Frederiksen ${ }^{2}$
}

(Received 28 January 2011; revised 26 September 2011)

\begin{abstract}
A new inverse method for determining the anomalous mean forcing functions responsible for climate change is presented. The method closes the mean field equations by representing the second order statistical moments as linear functions of the mean field. The coefficients of the linear parameterisation are determined by least-squares regression. The technique successfully reproduces the anomalous forcing functions responsible for the observed change in climate simulations between the periods July 1949-1968 and 1975-1994.
\end{abstract}

\section{Contents}

\section{Introduction}

http://journal .austms.org.au/ojs/index.php/ANZIAMJ/article/view/3930 gives this article, (c) Austral. Mathematical Soc. 2011. Published October 12, 2011. ISSN 1446-8735. (Print two pages per sheet of paper.) Copies of this article must not be made otherwise available on the internet; instead link directly to this URL for this article. 


\section{Inverse method}

3 The two level quasi-geostrophic equations

4 Numerical simulations

C830

5 Conclusion

C833

References

C835

\section{Introduction}

Studies, such as that by Frederiksen and Frederiksen [1], have shown that the mean circulation patterns of the atmosphere have been changing systematically over the latter half of the 20th century. Moreover, these changes in turn account for the reduction in storm development and rainfall observed over southern Australia and particularly over south-west of Western Australia during winter. It is therefore of considerable interest to establish what dynamical and physical processes are responsible for the change in the mean circulation patterns.

In typical (forward) climate model studies one simulates the response of the climate to a perturbation in known external forcing agents such as greenhouse gases and sea surface temperatures. However, in the climate change attribution problem one needs instead to determine the anomalous external forcing responsible for the observed changes in climate. In other words, an inverse model of climate change attribution is needed. This problem is complicated by the two way interaction of the large scale circulation and weather systems through heat and momentum fluxes, or potential vorticity fluxes, resulting in vacillation cycles of growth and decay [2]. At steady state the mean circulation is driven not only by the external forcing functions but also by the eddy fluxes, which appear as second order moments in the mean field equations. Formally, the problem is equivalent to the statistical 
dynamical closure problem of turbulence where determination of the moment of a given order requires knowledge of moments of higher order.

We present a method that closes the statistical equations for the climate by expressing the second order moments in terms of the mean field circulation. We are guided by the Quasi-diagonal Direct Interaction Approximation (QDIA) closure theory of Frederiksen [3] for the statistical dynamics of inhomogeneous flows. The QDIA closure has been successfully applied to many situations and shown to be in excellent agreement with direct numerical simulations (DNS) $[4,5]$. However, rather than solving the complex closure equations directly, here we close the equation for the mean climate using the DNS statistics to express the second order moments in terms of the mean field. As in the QDIA the second order moments are expressed as linear functions of the mean field but here the coefficients in the linear fit are obtained by least squares regression. Our approach is general and relatively simple to implement compared with solving the computationally demanding QDIA closure equations.

This article is structured as follows. Section 2 outlines our inverse modelling methodology in its most general form. Section 3 introduces the two level quasigeostrophic $(\mathrm{QG})$ model that is the basis of all the numerical experiments in this study. Section 4 presents some results on the simulation of the July 1949-1968 and 1975-1994 climate states with the two level QG model. We also apply the inverse model methodology to calculate the anomalous forcing function responsible for the shift in climate in the two periods. Finally, Section 5 summarises the results and presents our conclusions.

\section{Inverse method}

Let $\chi^{j}(\lambda, \mu, t)$ represent a prognostic field variable on the sphere. Here, the index $j$ represents discrete vertical levels of different fields such vorticity, divergence and temperature; $\lambda$ is the longitude; $\mu$ is the sine of the latitude; 
and $t$ represents time. The model that describes the evolution of the prognostic fields may also be horizontally discretized by expanding the fields in terms of spherical harmonics

$$
\chi^{j}(\lambda, \mu, t)=\sum_{m, n} x_{m n}^{j}(t) P_{n}^{m}(\mu) \exp (i m \lambda) .
$$

Here, $P_{n}^{m}(\mu)$ are Legendre functions; $m$ and $n$ are zonal and total wavenumbers, respectively; and $x_{m n}^{j}(t)$ are discretized spectral fields. Using equation (1), the prognostic equations for thermodynamical fields are written in the spectral form

$$
\begin{aligned}
\frac{\partial x_{m n}^{j}}{\partial t}= & \sum_{p, q, r, s, k, l}\left[\mathcal{K}^{j k l}(m, n ; p, q ; r, s) x_{-p q}^{k} x_{-r s}^{l}\right. \\
& \left.+\mathcal{A}^{j k l}(m, n ; p, q ; r, s) x_{-p q}^{k} \phi_{-r s}^{l}\right] \\
& -\sum_{k} D_{0}^{j k}(m, n) x_{m n}^{k}+f_{0}^{j}(m, n),
\end{aligned}
$$

where $\phi_{m n}^{j}$ is the spectral component of some spatially varying but time independent field $\phi^{j}(\lambda, \mu)$ such as the effective topography; $\mathcal{K}^{j k l}(m, n ; p, q ; r, s)$ and $\mathcal{A}^{\mathrm{jkl}}(\mathrm{m}, \mathrm{n} ; \mathrm{p}, \mathbf{q} ; \mathrm{r}, \mathrm{s})$ are interaction coefficients; $\mathrm{D}_{0}^{\mathrm{jk}}(\mathrm{m}, \mathrm{n})$ are the coefficients of the linear terms; and $f_{0}^{j}(m, n)$ is the forcing function.

Denoting time averaging at statistical steady state by angular brackets, from equation (2),

$$
\begin{gathered}
\sum_{p, q, r, s, k, l}\left[\mathcal{K}^{j k l}(m, n ; p, q ; r, s)\left\langle x_{-p q}^{k}\right\rangle\left\langle x_{-r s}^{l}\right\rangle\right. \\
\left.+\mathcal{A}^{j k l}(m, n ; p, q ; r, s)\left\langle x_{-p q}^{k}\right\rangle \phi_{-r s}^{l}\right] \\
+\left\langle T_{m n}^{j}\right\rangle-\sum_{k} D_{0}^{j k}(m, n)\left\langle x_{m n}^{k}\right\rangle+\left\langle f_{0}^{j}(m, n)\right\rangle=0, \\
\text { where }\left\langle T_{m n}^{j}\right\rangle=\sum_{p q} \sum_{r s} \sum_{k l} \mathcal{K}^{j k l}(m, n ; p, q ; r, s)\left\langle\hat{x}_{-p q}^{k} \hat{x}_{-r s}^{l}\right\rangle
\end{gathered}
$$


is the mean field nonlinear tendency due to the transient fields and we split $\chi_{m n}^{j}$ into mean, $\left\langle x_{m n}^{j}\right\rangle$, and transient, $\widehat{x}_{m \mathfrak{n}}^{j}$, parts.

The goal of the inverse climate model is to determine the mean forcing functions, $\left\langle\mathrm{f}_{0}^{j}(\mathrm{~m}, \mathrm{n})\right\rangle$, from the mean fields, $\left\langle x_{m \mathfrak{n}}^{j}\right\rangle$. However, equations (3) and (4) show that to determine the forcing functions not only are the mean fields required but also the second order moments $\left\langle\hat{x}_{-p q}^{k} \hat{x}_{-r s}^{l}\right\rangle$. This is the turbulence closure problem and we represent $\left\langle\mathrm{T}_{\mathrm{mn}}^{j}\right\rangle$ in the form indicated by the QDIA closure at steady state as a linear function of the mean field

$$
\left\langle T_{m n}^{j}\right\rangle=-\sum_{k} D_{t}^{j k}(m, n)\left\langle x_{m n}^{k}\right\rangle+f_{t}^{j}(m, n) .
$$

Here, $D_{t}^{j k}(m, n)$ and $f_{t}^{j}(m, n)$ (with subscript ' $t$ ' indicating transient contribution) are coefficients that we determine from the statistics of DNS as follows. We choose some appropriate initial state and perform a forward model simulation. The forcing function for this initial state may be approximated by some other procedure such as the iterative one described in Section 4. We then perturb this forcing function to yield a set of perturbed mean fields, $\left\langle\chi_{\mathrm{mn}}^{j}\right\rangle_{s}$, and their associated nonlinear mean field tendencies, $\left\langle\mathrm{T}_{\mathrm{mn}}^{j}\right\rangle_{\mathrm{s}}$, where $\mathrm{s}$ denotes distinct perturbations of the original climate state. Linear regression then determines the coefficients $D_{t}^{j k}(m, n)$ and $f_{t}^{j}(m, n)$ from this set of perturbed mean fields and mean field tendencies according to equation (5).

Using equations (3), (4), and (5), the climate model is written

$$
\begin{aligned}
& \sum_{p, q, r, s, k, l}\left[\mathcal{K}^{j k l}(m, n ; p, q ; r, s)\left\langle x_{-p q}^{k}\right\rangle\left\langle x_{-r s}^{l}\right\rangle\right. \\
&\left.+\mathcal{A}^{j k l}(m, n ; p, q ; r, s)\left\langle x_{-p q}^{k}\right\rangle \phi_{-r s}^{l}\right] \\
&-\sum_{k} D_{r}^{j k}(m, n)\left\langle x_{m n}^{k}\right\rangle+\left\langle f_{r}^{j}(m, n)\right\rangle=0 \\
& \text { where } \quad D_{r}^{j k}(m, n)=D_{0}^{j k}(m, n)+D_{t}^{j k}(m, n) \\
& \text { and } \quad\left\langle f_{r}^{j}(m, n)\right\rangle=\left\langle f_{0}^{j}(m, n)\right\rangle+f_{t}^{j}(m, n) .
\end{aligned}
$$


Here the subscript ' $r$ ' indicates that the coefficient is renormalised (or redefined). Unlike equation (3), equation (6) is statistically closed in the sense that only the mean field is required to calculate the mean forcing and not higher order moments. We examine the performance of equation (6) in relation to equation (3) to see how well the nonlinear mean field tendencies are represented by the linear closure model. We expect that as long as the climate state in question is sufficiently close to the climate state used to calculate the linear regression coefficients then the linear closure would indeed be successful. The question of how far away in phase space the new climate state can be is addressed by experimentation in subsequent sections.

\section{The two level quasi-geostrophic equations}

We apply the climate change attribution methodology to the two level quasigeostrophic model of Frederiksen [6] that was also employed in the subgrid modelling study of Zidikheri and Frederiksen [7]. For flow on the spherical earth these equations take the form

$$
\frac{\partial q^{j}}{\partial t}=-J\left(\psi^{j}, q^{j}+h^{j}\right)-2 \frac{\partial \psi^{j}}{\partial \lambda}-\left[\alpha^{j}+(-1)^{\rho} \gamma_{0}^{j} \nabla^{2 \rho}\right] \zeta^{j}+\kappa\left(\tilde{q}^{j}-q^{j}\right),
$$

where $j=1$ is the upper level and $j=2$ is the lower level. The (reduced) potential vorticity at level $\boldsymbol{j}$ is defined as

$$
q^{j}=\nabla^{2} \psi^{j}+(-1)^{j} F_{L}\left(\psi^{1}-\psi^{2}\right),
$$

where $\psi^{j}$ are level dependent streamfunctions, $\zeta^{j}=\nabla^{2} \psi^{j}$ are relative vorticities, and $F_{L}$ is the layer coupling parameter. The latter is related to the internal Rossby radius of deformation $r_{\text {int }}=\left(2 F_{L}\right)^{-1 / 2}$. The model includes drag specified by $\alpha^{j}$ and prescribed viscosities $v_{0}^{j} ; \rho$ is a positive integer that describes the order of the Laplacian operator. The equations are nondimensional, with the earth's radius as length scale and the inverse of earth's 
angular velocity as a time scale. The effective topography, which is independent of time, is represented by $h^{j}=2 \mu \mathrm{H}^{j} / \mathrm{H}_{0}$, where $\mathrm{H}^{j}$ is the topographic height and $\mathrm{H}_{0}$ is a scale height. We also allow for a simple representation of the effects of heating through a specification of the field, $\tilde{\mathrm{q}}^{j}$, and dissipation, $k$, that controls how rapidly the flow is being relaxed towards this field. The parameterized heating creates a mean vertical shear in the flow that results in the formation of energetic eddies which are model representations of mid-latitude weather systems.

We work in the spectral domain, and the spectral model is obtained by expanding the fields $\psi^{j}, \zeta^{j}, q^{j}$ and $h^{j}$ in terms of spherical harmonics, as in equation (1):

$$
\begin{aligned}
\frac{\partial q_{m n}^{j}}{\partial t}= & \sum_{p, q, r, s} A(m, n ; p, q ; r, s)\left[\zeta_{-p q}^{j} q_{-r s}^{j}+\zeta_{-p q}^{j} h_{-r s}^{j}\right] \\
& -d_{0}^{j}(m, n) \zeta_{m n}^{k}+k\left(\tilde{q}_{m n}^{j}-q_{m n}^{j}\right) .
\end{aligned}
$$

Here,

$$
\begin{aligned}
A(m, n ; p, q ; r, s)= & -i[q(q+1)]^{-1} \\
& \times \int_{-1}^{1} d \mu P_{n}^{m}(\mu)\left[p P_{q}^{p}(\mu) \frac{d}{d \mu} P_{s}^{r}(\mu)-r P_{s}^{r}(\mu) \frac{d}{d \mu} P_{q}^{p}(\mu)\right] .
\end{aligned}
$$

In equations (11) and (12), the interaction coefficient vanishes unless the selection rules $\mathrm{m}+\mathrm{p}+\mathrm{r}=0, \mathrm{n}+\mathrm{q}+\mathrm{s}=$ odd integer, and $|\mathrm{q}-\mathrm{s}|<\mathrm{n}<\mathrm{q}+\mathrm{s}$ are satisfied. The real part of the complex operator $d_{0}^{j}$ describes horizontal dissipation and its imaginary part describes the frequency of Rossby waves

$$
d_{0}^{j}(m, n)=\alpha^{j}+v^{j}[n(n+1)]^{\rho}-i \frac{2 m}{n(n+1)} .
$$

Equation (11) is cast in the more general form of equation (2) by identifying $\chi^{j}$ with $q^{j}$ and identifying $\phi^{j}$ with $h^{j}$ and limiting the indices to $j, k, l=1,2$ in 
equation (2). Additionally,

$$
\mathcal{A}^{j k l}(m, n ; p, q ; r, s)=a^{j k} \delta^{j l} A(m, n ; p, q ; r, s)
$$

and

$$
\mathcal{K}^{\mathrm{jkl}}(\mathrm{m}, \mathrm{n} ; \mathrm{p}, \mathrm{q} ; \mathrm{r}, \mathrm{s})=\frac{1}{2}\left[\mathcal{A}^{j \mathrm{kl}}(\mathrm{m}, \mathrm{n} ; \mathrm{p}, \mathrm{q} ; \mathrm{r}, \mathrm{s})+\mathcal{A}^{\mathrm{jlk}}(\mathrm{m}, \mathrm{n} ; \mathrm{r}, \mathrm{s} ; \mathrm{p}, \mathrm{q})\right] .
$$

Here, $\delta^{j l}$ is the Kronecker delta function, and

$$
a^{11}=a^{22}=\frac{q(q+1)+F_{L}}{q(q+1)+2 F_{L}} \quad \text { and } \quad a^{12}=a^{21}=\frac{F_{L}}{q(q+1)+2 F_{L}} .
$$

Moreover, the matrix dissipation operator is $D_{0}^{j k}=d_{0}^{j} a^{j k}+k \delta^{j k}$ and the bare forcing is $f_{0}^{j}(m, n)=\kappa \tilde{q}_{m n}^{j}$.

\section{Numerical simulations}

Firstly, we generate data by integrating equation (11) forward in time to a statistical steady state using prescribed parameters and relaxation fields, $\tilde{\mathbf{q}}_{\mathrm{mn}}^{j}$. We run two simulations with different values of $\tilde{\mathbf{q}}_{\mathrm{mn}}^{j}$ corresponding to the observed climatologies of the periods 1949-1968 and 1975-1994. We iteratively nudge the simulation climatologies towards the observed climatologies starting with the relaxation fields set equal to the observed climatologies. For subsequent iterations the relaxation fields are calculated as

$$
\left[\tilde{\mathbf{q}}_{m n}^{j}\right]_{i}=\left[\tilde{\mathbf{q}}_{m n}^{j}\right]_{i-1}+\left\langle\left[\mathbf{q}_{m n}^{j}\right]_{o b s}\right\rangle-\left\langle\left[\mathbf{q}_{m n}^{j}\right]_{i-1}\right\rangle
$$

with subscript $i$ representing the iteration step and subscript "obs" indicating the observed fields. We choose $i=22$ to be the final iteration step. Other chosen parameters are: $\left[\alpha^{1}\right]^{-1}=20$ days and $\left[\alpha^{2}\right]^{-1}=5$ days; $v^{1}=v^{2}=$ $1.55 \times 10^{16} \mathrm{~m}^{4} \mathrm{~s}^{-1}$ and $\rho=2 ; \mathrm{k}^{-1}=10$ days; $\mathrm{F}_{\mathrm{L}}=3.125 \times 10^{-12} \mathrm{~m}^{-2}$. Global 


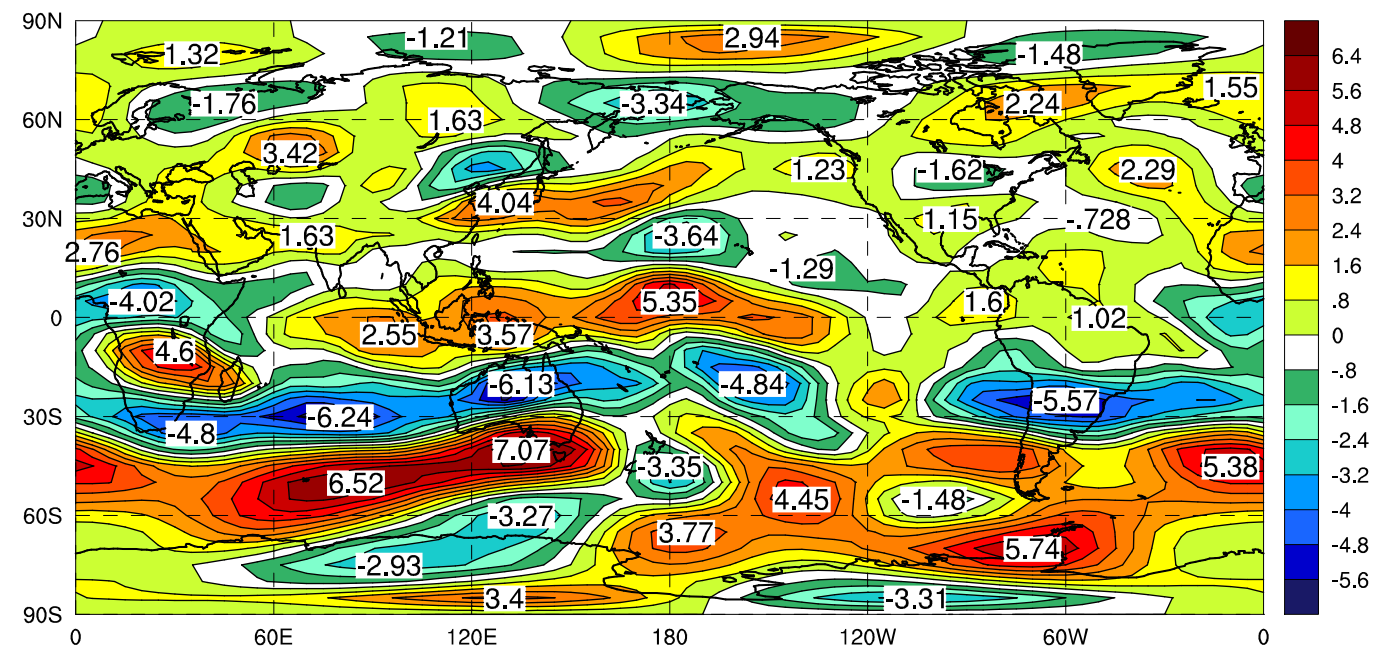

Figure 1: Differences between barotropic zonal winds (in $\mathrm{ms}^{-1}$ ) in the July 1975-1994 and July 1949-1968 climate simulations.

topographic data at the resolution of T15 is used in the lower level with a scale height $\mathrm{H}_{0}=8 \mathrm{~km}$. The model resolution is T31.

The results are presented in terms of the barotropic (vertically averaged) flow instead of the flow at Levels 1 and 2. The differences in the time average of the barotropic flow field between the two climate periods, 1975-1994 and 19491968, are shown in Figure 1. In the southern hemisphere the barotropic zonal winds are reduced in midlatitudes and enhanced poleward. Figure 3(a) shows the corresponding barotropic zonal wind forcing function differences. Our goal is to reproduce the forcing function differences in Figure 3(a) responsible for the climate differences in Figure 1 using the inverse model proposed in the previous sections. Figure 2(a) shows the differences in the nonlinear tendency $\left\langle\mathrm{T}_{\mathrm{mn}}^{\mathrm{j}}\right\rangle$ in physical space and scaled by $\mathrm{k}^{-1}$ between the two periods. This is the field that must be parameterized in terms of the mean field so that the forcing functions can be computed directly. It describes the contribution of the transient fields to the mean (mainly large scale) fields. 
(a)

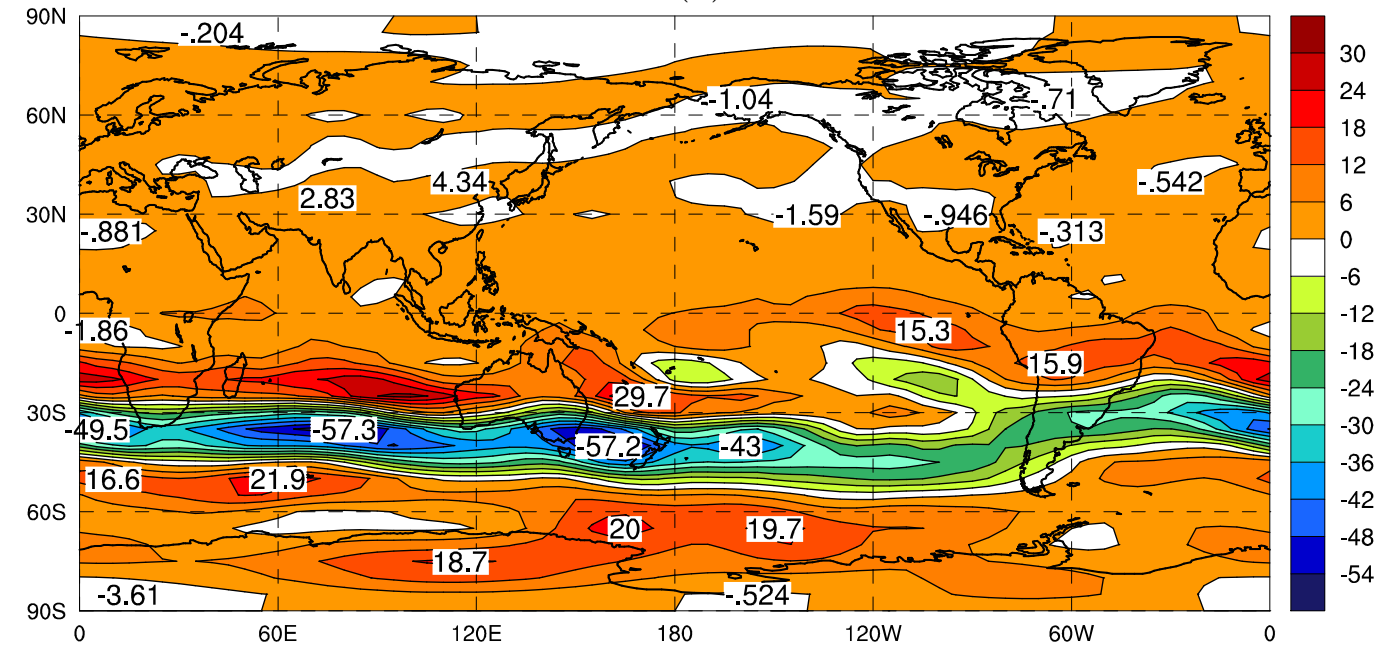

(b)

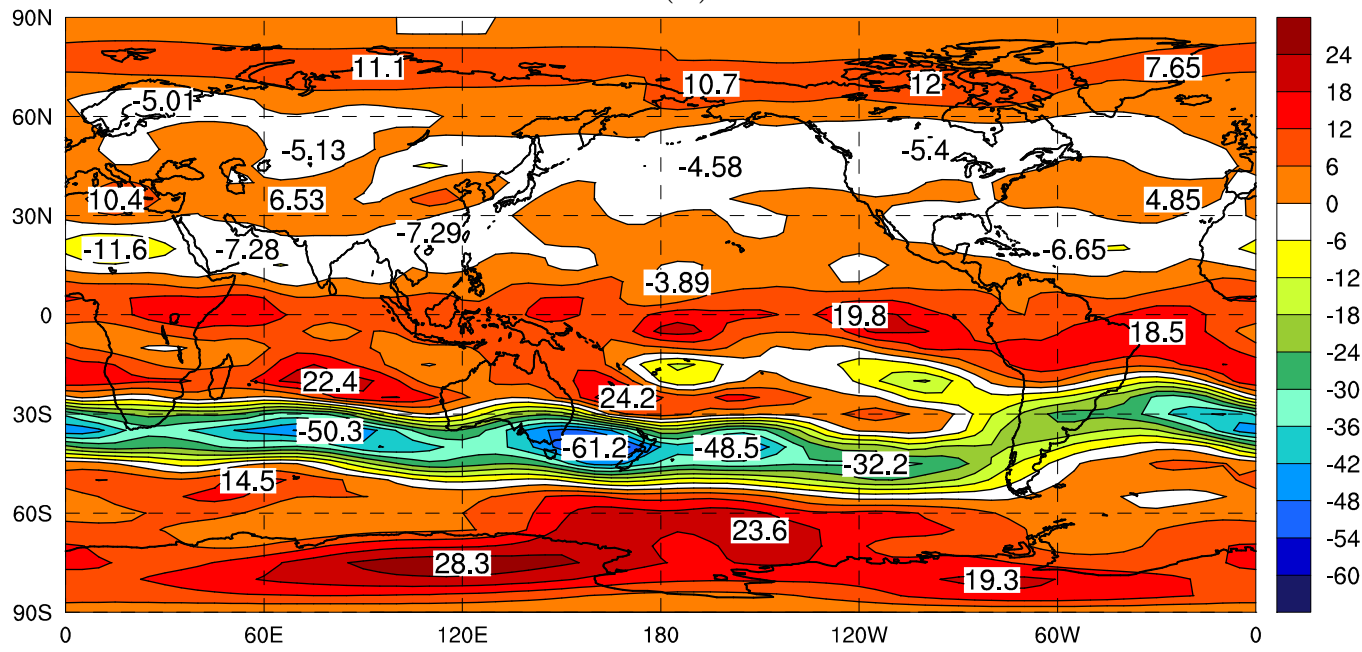

Figure 2: Differences between barotropic zonal wind nonlinear tendencies (in $\mathrm{m} \mathrm{s}^{-1}$ and scaled by $\mathrm{K}^{-1}$ ) in the July 1975-1994 and July 1949-1968 climate simulations as obtained from (a) numerical simulations and (b) linear regression. 
To calculate the model response to anomalous forcing we generate an ensemble of ten perturbed model climates by perturbing the relaxation fields. The perturbed relaxation fields $\left[\tilde{\mathfrak{q}}_{\mathrm{mn}}^{j}\right]_{\mathrm{p}}$ are obtained by linearly combining the

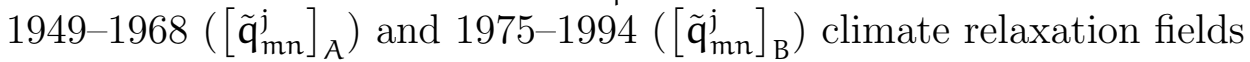

$$
\left[\tilde{\mathbf{q}}_{m n}^{j}\right]_{s}=\gamma_{s}\left[\tilde{\mathbf{q}}_{m n}^{j}\right]_{A}+\left(1-\gamma_{s}\right)\left[\tilde{\mathbf{q}}_{m n}^{j}\right]_{B} .
$$

The parameter $\gamma_{s}$ is chosen to have the range $[0.525,0.75]$ so that all the perturbed climates are closer to the earlier (1949-1968) climate than to the later (1975-1994) climate. This choice is motivated by the fact that we want to use the later climate as a test case for evaluating the performance of our inverse model. Choosing perturbed climate states closer to the earlier climate ensures that the results are not biased towards the later climate. Figure 2(b) shows the mean field nonlinear tendency differences between the periods 1949-1968 and 1975-1994 reconstructed using equation (5) after obtaining the coefficients $D_{t}^{j k}(m, n)$ and $f_{t}^{j}(m, n)$ from the perturbed states. Comparing this figure to Figure 2(a), see that the linear approximation does indeed work quite well, with most large scale structures being reproduced successfully. Finally, Figure 3(b) shows the reconstructed difference (1975-1994 and 1949-1968) in forcing functions using the parameterized form of $\left\langle\mathrm{T}_{m n}^{j}\right\rangle$. The reconstructed anomalous forcing function is in good agreement with the actual anomaly used in the numerical climate simulation (Figure 3(a)).

\section{Conclusion}

We proposed a scheme to close the equations describing climate at the first order by parametrising the nonlinear transient field term as a term linear in the mean fields. In this scheme, the coefficients required in the linear model are determined by linearly regressing an ensemble of perturbed climate states. We found that the time average of the nonlinear term in the numerical climate simulations is qualitatively well reproduced by this method. The utility of this method is that the anomalous forcing function responsible for an observed 
(a)

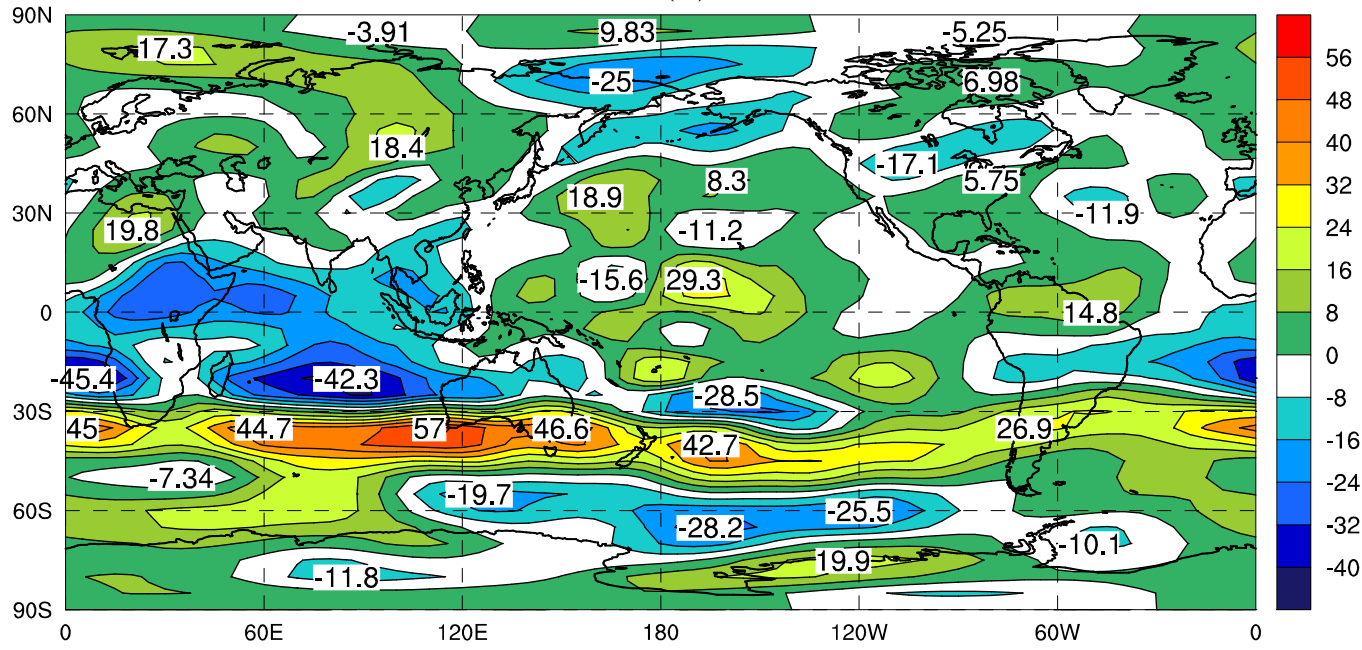

(b)

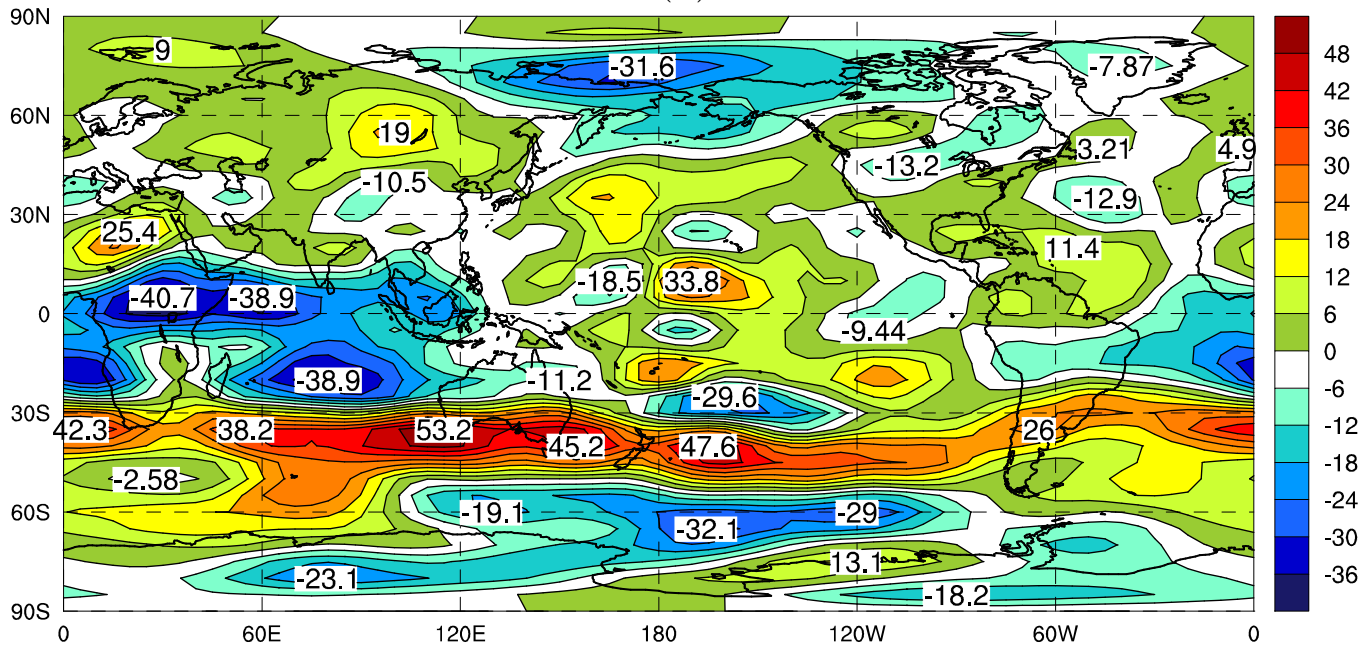

Figure 3: Differences between barotropic zonal wind forcing functions (in $\mathrm{m} \mathrm{s}^{-1}$ and scaled by $\mathrm{K}^{-1}$ ) in the July 1975-1994 and July 1949-1968 climate simulations used in (a) numerical simulations and (b) obtained from the inverse model. 
shift in a climate state can be directly calculated from the climate states without an explicit knowledge of the second order moments that would be required in a fully nonlinear model. Such an efficient inverse model is of great use in the problem of attributing climate change to a variety of physical and dynamical processes. In future studies we aim to apply the method to more complex climate models such general circulation models.

Acknowledgements The first author is supported by the Indian Ocean Climate Initiative program.

\section{References}

[1] J. S. Frederiksen and C. S. Frederiksen. Interdecadal changes in southern hemisphere winter storm track modes. Tellus, 59A:599-617, 2007. doi:10.1111/j.1600-0870.2007.00264.x C824

[2] J. S. Frederiksen. Growth and vacillation cycles of disturbances in southern hemisphere flows. J. Atmos. Sci., 38:1360-1375, 1981. doi:10.1175/1520-0469(1981)038;1360:GAVCOD ¿2.0.CO;2 C824

[3] J. S. Frederiksen. Subgrid-scale parameterizations of eddy-topographic force, eddy viscosity, and stochastic backscatter for flow over topography. J. Atmos. Sci., 56:1481-1494, 1999. doi:10.1175/1520-0469(1999)056¡1481:SSPOET ¿2.0.CO;2 C825

[4] T. J. O'Kane and J. S. Frederiksen. The QDIA and regularized QDIA closures for inhomogeneous turbulence over topography. J. Fluid. Mech., 504:133-165, 2004. doi:10.1017/S0022112004007980 C825

[5] J. S. Frederiksen and T. J. O'Kane. Inhomogeneous closure and statistical mechanics for Rossby wave turbulence over topography. $J$. Fluid. Mech., 539:137-165, 2005. doi:10.1017/S0022112005005562 C825 
[6] J. S. Frederiksen. Precursors to blocking anomalies: The tangent linear and inverse problems. J. Atmos. Sci., 55:2419-2436, 1998. doi:10.1175/1520-0469(1998)055;2419:PTBATT¿2.0.CO;2 C828

[7] M. J. Zidikheri and J. S. Frederiksen. Stochastic subgrid parameterizations for simulations of atmospheric baroclinic flows. $J$. Atmos. Sci., 66:2844-2858, 2009. doi:10.1175/2009JAS3036.1 C828

\section{Author addresses}

1. Meelis J. Zidikheri, Centre for Australian Weather and Climate Research, Bureau of Meteorology, Docklands, Victoria, Australia. mailto:m.zidikheri@bom.gov.au

2. Jorgen S. Frederiksen, Climate Adaptation Flagship, CSIRO Marine and Atmospheric Research, Aspendale, Victoria, Australia 